\title{
Distribución de patrones PRA en aislamientos clínicos del complejo Mycobacterium avium procedentes de España y Suramérica
}

\author{
Martha Isabel Murcia ${ }^{1, *}$, Sylvia Cardoso Leao ${ }^{2}$, Viviana Ritacco ${ }^{3}$, Elia Palenque ${ }^{6}$, \\ Rosangela Siqueira de Oliveira ${ }^{2}$, Ana Reniero ${ }^{3}$, Maria Carmen Menendez ${ }^{1, \# \text {, }}$ \\ María Alice da Silva Telles ${ }^{4}$, David Jamil Hadad ${ }^{5}$, Lucía Barrera ${ }^{3}$, María Jesús García ${ }^{1}$ \\ ${ }^{1}$ Departamento de Medicina Preventiva, Facultad de Medicina, Universidad Autónoma, Madrid, España. \\ 2 Departamento de Microbiologia, Imunologia e Parasitologia, Universidade Federal de São Paulo, Escola \\ Paulista de Medicina, São Paulo, Brasil. \\ ${ }^{3}$ Instituto Nacional de Enfermedades Infecciosas, ANLIS "Carlos Malbrán" y Consejo Nacional de \\ Investigaciones Científicas, Buenos Aires, Argentina. \\ ${ }^{4}$ Setor de Micobactérias, Instituto Adolfo Lutz, São Paulo, Brasil. \\ ${ }^{5}$ Núcleo de Doenças Infecciosas, Universidade Federal do Espírito Santo, Espírito Santo, Brasil. \\ ${ }^{6}$ Laboratorio de Micobacterias, Servicio de Microbiología, Hospital 12 de Octubre, Madrid, España. \\ * Departamento de Microbiología, Facultad de Medicina, Universidad Nacional, Bogotá, Colombia. \\ \# Division of Mycobacterial Research, NIMR, London, United Kingdom. \\ La infección por el complejo Mycobacterium avium (MAC) es la infección sistémica más frecuente \\ en la fase terminal del SIDA. Las sondas de ADN disponibles en el mercado para la identificación \\ de micobacterias son muy precisas pero extremadamente costosas. Por eso, la mayoría de los \\ laboratorios clínicos de Latinoamérica aún tipifican micobacterias mediante pruebas fenotípicas \\ que son lentas, laboriosas y poco precisas. En este trabajo se aplicó el análisis del polimorfismo \\ de los fragmentos de restricción del gen hsp65 (PRA) a la identificación de MAC en 163 \\ aislamientos clínicos procedentes de España y Suramérica. El genotipo PRA predominante en \\ cada país fue: M. avium tipo I en Argentina (23/42, 55\%) y Brasil (48/72, 67\%), M. avium tipo II \\ en España (18/26, 69\%) y M. avium tipo III en Colombia (10/23, 43\%). Este último genotipo, que \\ aún no fue descrito fuera del continente americano, resultó muy infrecuente en los otros tres \\ países del estudio. Se discuten ventajas e inconvenientes de la aplicación del PRA al diagnóstico \\ micobacteriológico.
}

Palabras clave: complejo Mycobacterium avium, gen hsp65, patrones PRA, Suramérica, España, M. avium tipo III

\section{Distribution of PRA patterns of clinical isolates of the Mycobacterium avium complex from Spain and South America}

Mycobacterium avium complex (MAC) infections are the most frequent systemic infections associated with advanced AIDS. DNA probes for accurate identification of mycobacteria are available but are very expensive in many Latin American settings. Consequently, most Latin American diagnostic laboratories employ inaccurate and outdated tests for mycobacteria identification. Therefore, PCR restriction analysis (PRA) of the $h s p 65$ gene was evaluated for the identification of 163 MAC human isolates originated from Spain and South America. The predominant PRA type in each country was: $M$. avium type I in Argentina $(23 / 42,55 \%)$ and Brazil (48/72, 67\%), M. avium type II in Spain (18/26, 69\%) and M. avium type III in Colombia (10/ $23,43 \%)$. The Colombia frequency is noteworthy, since the PRA type III was quite infrequent in the other three countries. Furthermore, its presence has not been reported outside the Americas. The advantages and disadvantages of PRA in diagnostic mycobacteriology are discussed.

Key words: Mycobacterium avium complex, hsp65 gene, PRA patterns, South America, Spain, M. avium type III. 
Desde la emergencia de la pandemia de sida, la infección por el complejo Mycobacterium avium se convirtió en la infección sistémica más frecuente en la fase terminal de la enfermedad $(1,2)$. El complejo M. avium comprende dos especies claramente aceptadas, Mycobacterium avium y Mycobacterium intracellulare así como otras micobacterias de difícil asignación (3). De acuerdo con las características fenotípicas y genotípicas, unido a la virulencia y al rango de hospedadores, se han reconocido cuatro subespecies de M. avium: M. avium, subespecie avium; $M$. avium, subespecie hominissuis; $M$. avium, subespecie paratuberculosis, y M. avium, subespecie silvaticum (4). Hasta el momento, no se ha descrito la existencia de subespecies en M. intracellulare.

Las pruebas fenotípicas, las únicas accesibles a gran parte de los laboratorios de diagnóstico clínico en Latinoamérica, permiten la identificación del complejo $M$. avium, pero no distinguen entre especies o subespecies dentro del complejo. Además, especies tan distintas como Mycobacterium lentiflavum y Mycobacterium simiae pueden ser erróneamente clasificadas como complejo $M$. avium por pruebas fenotípicas. La correcta identificación de los distintos miembros del complejo M. avium permitió recientemente confirmar que $M$. avium es causante de infección diseminada en pacientes con sida (3), pero aún no se ha estudiado en detalle la relación de infección diseminada con $M$. intracellulare.

La sonda comercial de hibridación (AccuProbe system; GenProbe Inc., San Diego, CA) $(5,6)$ se considera la prueba de referencia para la identificación rápida de miembros del complejo M. avium y es ampliamente utilizada en los países desarrollados. Pero para lograr la identificación de especie se deben aplicar tres sondas diferentes, lo que, añadido a su elevado costo, hace difícil

Correspondencia:

María Jesús García, Departamento de Medicina Preventiva, Facultad de Medicina, Universidad Autónoma, C/ Arzobispo Morcillo, 4, 28029, Madrid, España.

Teléfono: +34-91.3975440; fax: +34-91.3975353.

mariaj.garcia@uam.es

Recibido: 30/06/04; aceptado: 10/02/04 su utilización en los países de Latinoamérica. Telenti y colaboradores propusieron un método de identificación de micobacterias basado en el análisis del polimorfismo de los fragmentos obtenidos al digerir el gen hsp65 con enzimas de restricción ( $P C R$ restriction enzyme analysis, PRA) (7). Este método ofrece varias ventajas para los laboratorios clínicos: es más rápido que la identificación fenotípica, es relativamente fácil y resulta mucho menos costoso que la sonda de hibridación comercial (8).

Se han descrito varios patrones PRA dentro del complejo $M$. avium: 4 variantes para $M$. avium $(9,10)$ y 6 para $M$. intracellulare $(5,11)$. La variante I de $M$. avium se ha encontrado con mayor frecuencia asociada con infección diseminada en pacientes con sida mientras que las variantes II y III han sido descritas con mayor frecuencia en muestras ambientales (11). Estos hallazgos sugieren que las variantes PRA de $M$. avium pueden diferir en cuanto a su virulencia y transmisibilidad (12).

Con el fin de evaluar la utilidad del método de PRA en la identificación de aislamientos del complejo $M$. avium de origen humano y analizar la distribución geográfica de las variantes del complejo, se planteó analizar por este método aislamientos clínicos procedentes de cuatro países.

Se incluyeron 163 aislamientos clínicos pertenecientes al complejo $M$. avium originarios de Brasil $(n=72)$, Argentina $(n=42)$, Colombia $(n=23)$ y España $(n=26)$. Las muestras se obtuvieron en hospitales y centros de diagnóstico especializados en el cultivo de micobacterias. Si bien se analizó más de una muestra de algunos pacientes, para este estudio se consideró un solo aislamiento por paciente. Los aislamientos analizados en Latinoamérica corresponden al total de casos del complejo $M$. avium confirmados bacterio-lógicamente en cada institución, y recibidos durante los períodos estudiados. Los aislamientos identificados en el Instituto Adolfo Lutz de São Paulo provenían de centros de referencia de sida y centros de salud del estado de São Paulo. Los aislamientos remitidos para su identificación al Instituto Nacional de Enfermedades Infecciosas de Buenos Aires 
provenían de 15 hospitales de diferentes regiones de Argentina. Los aislamientos identificados en el Instituto Nacional de Salud de Bogotá procedían de 3 hospitales de referencia ubicados en Bogotá, D.C., que reciben el mayor número de pacientes con sida de Bogotá y de otras regiones del país. En Colombia, el análisis comprende dos muestras estudiadas en dos períodos distantes en el tiempo, como se refleja en el cuadro $1(2,13)$. Los aislamientos identificados en el Hospital 12 de Octubre de Madrid procedían de 3 hospitales que atienden habitantes de esa ciudad. La identificación de los aislamientos como miembros del complejo se hizo mediante pruebas fenotípicas o sondas comerciales de ADN.

Para la determinación de los patrones PRA de estos aislamientos clínicos, se empleó el método descrito por Telenti y colaboradores (7). Brevemente, el ADN se obtuvo mediante hervido de una suspensión bacteriana (10). El segmento específico del gen hsp65 se amplificó mediante PCR y el producto amplificado de 440 pares de bases se sometió a restricción enzimática con BstE II y Hae III. La identificación se realizó por determinación de los tamaños de los fragmentos de digestión según algoritmos de referencia (8, PRASITE: www.hospud.ch.8005).

El algoritmo para la identificación mediante PRA supone actualmente un problema, dado que la nomenclatura de los patrones es confusa o contradictoria entre las distintas fuentes de información accesibles a los usuarios. Por ejemplo, PRASITE, la única base de datos de acceso libre, invierte el orden descrito originalmente en la literatura entre patrones PRA de M. avium, tipo I y II $(7,9,11)$. Estas confusiones pueden inducir a errores en la evaluación de la frecuencia de los diferentes tipos PRA y resaltan la necesidad de una revisión y actualización frecuente de las bases de datos (14). En el presente trabajo se han identificado los patrones según fueron descritos en las referencias originales $(5,7,9,10)$. Por tanto, las variantes I y II de $M$. avium tienen sus patrones intercambiados respecto a lo que indica la base de datos PRASITE (figura 1).

Entre los aislamientos identificados como complejo M. avium en este trabajo, se detectaron

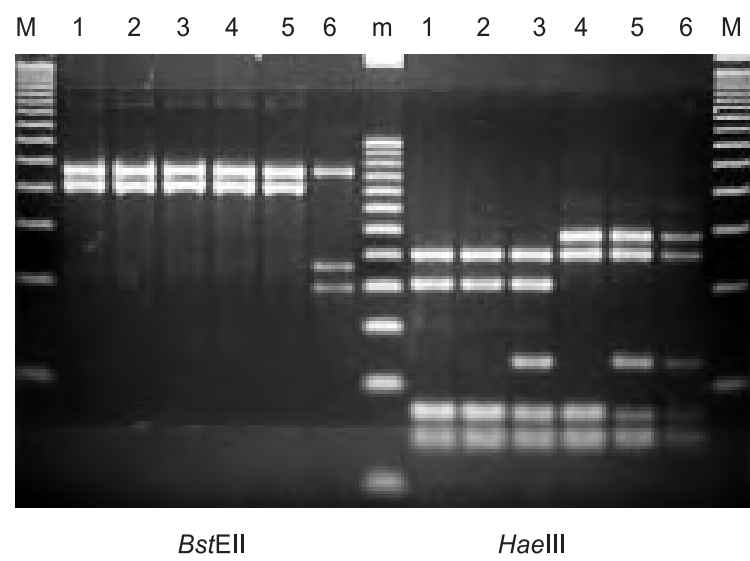

Figura 1. Digestión del producto amplificado del gen $h s p 65$ con BstEll y Haell. Carril 1: M. avium ATCC 25291; carril 2: M. avium I; carril 3: $M$. avium II; carril 4: $M$. avium III; carril 5: $M$. avium IV; carril 6: $M$. intracellulare ATCC 13950; carril M: 50 bp DNA ladder (Gibco/BRL); carril m: 25 bp DNA ladder (Gibco/BRL).

cuatro variantes PRA de $M$. avium y una variante de $M$. intracellulare (figura 1). Se detectó, además, un patrón M. lentiflavum IV (cuadro 1). Debe señalarse que esta última especie es muy similar al complejo M. avium por pruebas bioquímicas.

La mayoría de los patrones obtenidos correspondió a las diferentes variantes de $M$. avium (porcentaje medio de $89,5 \%$ ). Sólo en Argentina, y entre pacientes VIH negativos, se aisló una mayor proporción de $M$. intracellulare.

Cuando se compararon los dos patrones mayoritarios de M. avium (M. avium, tipos I y II), el primero apareció con más frecuencia en pacientes positivos para VIH y en los países suramericanos (porcentaje medio del 54\%); el segundo fue el más frecuente en la muestra española. El origen geográfico también se relacionó con el patrón más frecuente; así, Argentina y Brasil presentaron mayoritariamente M. avium, tipo I ( $55 \%$ y $67 \%$, respectivamente); España presentó un nivel mayor del tipo II (69\%) y el tipo III fue el más frecuente en la muestra colombiana (43\%). La elevada proporción de esta variante en los aislamientos de Colombia es sorprendente, dado que se trata de un patrón que sólo ha sido descrito excepcionalmente $(9,11)$ y sólo en muestras aisladas en el continente americano. 
Cuadro 1. Distribución de patrones PRA en 163 aislamientos clínicos del complejo Mycobacterium avium originarios de Suramérica y España.

\begin{tabular}{|c|c|c|c|c|c|c|c|c|c|}
\hline & & \multirow[b]{3}{*}{ Año } & \multicolumn{6}{|c|}{ Patrón PRA n (\%) } & \multirow{3}{*}{ m } \\
\hline & & & \multicolumn{4}{|c|}{$\begin{array}{c}\text { M. } \\
\text { avium }\end{array}$} & \multirow{2}{*}{$\frac{M .}{\text { intracellulare }}$} & \multirow{2}{*}{$\frac{\begin{array}{c}M . \\
\text { lentiflavum }\end{array}}{\text { IV }}$} & \\
\hline & & & I & II & III & IV & & & \\
\hline \multicolumn{10}{|c|}{ Colombia $(n=23)$} \\
\hline & $\mathrm{VIH}+$ & 1995 & 3 & 2 & 0 & 0 & 0 & 0 & 5 \\
\hline & VIH - & & 0 & 0 & 0 & 0 & 0 & 0 & 0 \\
\hline & $\mathrm{VIH}+$ & 2000 & 6 & 0 & 9 & 0 & 2 & 0 & 17 \\
\hline & VIH - & & 0 & 0 & 1 & 0 & 0 & 0 & 1 \\
\hline \multicolumn{10}{|c|}{ Brasil ( $n=72)$} \\
\hline & $\mathrm{VIH}+$ & $1995-1998$ & 39 & 12 & 2 & 0 & 1 & 1 & 55 \\
\hline & Desconocido & & 9 & 6 & 0 & 0 & 2 & 0 & 17 \\
\hline \multicolumn{10}{|c|}{ Argentina $(n=42)$} \\
\hline & $\mathrm{VIH}+$ & 2002 & 19 & 3 & 1 & 0 & 3 & 0 & 26 \\
\hline & VIH - & & 2 & 0 & 0 & 0 & 10 & 0 & 12 \\
\hline & Desconocido & & 2 & 1 & 0 & 1 & 0 & 0 & 4 \\
\hline \multicolumn{10}{|c|}{ España $(n=26)$} \\
\hline & $\mathrm{VIH}+$ & $1998-2001$ & 3 & 4 & 0 & 0 & 0 & 0 & 7 \\
\hline & $\mathrm{VIH}-$ & & 1 & 7 & 0 & 0 & 0 & 0 & 8 \\
\hline & Desconocido & & 3 & 7 & 0 & 0 & 1 & 0 & 11 \\
\hline Total & & & $87(53,4)$ & $42(25,7)$ & $13(8,0)$ & $1(0,6)$ & $19(11,7)$ & $1(0,6)$ & $63(100)$ \\
\hline
\end{tabular}

Las muestras colombianas están distribuidas en dos periodos de aislamiento que se corresponden con el periodo anterior a la instauración de la terapia múltiple antirretroviral en los pacientes con infección HIV (1995) o posterior a la misma (2000). Al pie de cada columna, se indica el número total de aislamientos para cada tipo de variante PRA y el porcentaje que representa entre paréntesis.

Cuando se compararon entre sí los resultados obtenidos en las dos muestras de Colombia, la distribución de los patrones PRA resultó llamativamente diferente. El patrón de M. avium, tipo III, recién apareció en la segunda muestra, es decir, después de la instauración de la terapia múltiple antirretroviral en la infección por VIH (highly active anti-retroviral therapy, HAART) (cuadro 1). La posible relación entre M. avium, tipo III, y HAART requiere un estudio más detallado.

Con cierta frecuencia se han encontrado patrones de restricción PRA compatibles con el complejo $M$. avium en aislamientos que resultaron negativos para las tres sondas comerciales o únicamente positivos para la sonda de complejo pero negativos para las dos sondas específicas de especie. Durante la ejecución de este estudio, detectamos cinco aislamientos de Colombia y otros cinco de España con esta característica (8 dieron M. avium, tipo I, y 2, M. intracellulare, tipo I) que no se incluyeron en el análisis. Estos aislamientos podrían identificarse como complejo $M$. avium "other" según la descripción de Smole y colaboradores (11). Su aparición determina los límites de confianza del método de PRA que, así como permite detectar errores de identificación por métodos bioquímicos, como hemos encontrado en el caso de $M$. lentiflavum, también puede conducir a un diagnóstico erróneo si se utiliza como único método de identificación. Es importante notar que especies como M. simiae y $M$. lentiflavum se identifican como complejo $M$. avium por métodos fenotípicos y presentan perfiles de PRA semejante a los descritos para $M$. avium III y para $M$. intracellulare I, respectivamente. La identificación definitiva de dichas especies depende de la realización de otras pruebas moleculares o bioquímicas.

En síntesis, el método de PRA constituye una valiosa herramienta diagnóstica que puede considerarse como una prueba de orientación y 
apoyo a la identificación en un laboratorio de micobacterias, además de ser relativamente fácil y de costo poco elevado. Aunque la identificación de especies micobacterianas no debe basarse en una sola prueba, no existe por el momento una metodología que presente un balance costobeneficio tan favorable en este campo como el PRA.

\section{Agradecimientos}

M. I. Murcia se encuentra adelantando trabajo doctoral con subvención de la Universidad Nacional (Bogotá, Colombia). Los laboratorios participantes reciben financiación de la Comunidad Europea, programa INCO-DEV (ICA4-CT-200110087) y todos son miembros de RELACTB (Red Europea Latinoamericana y del Caribe para el Estudio de la Tuberculosis).

\section{Referencias}

1. Coker RJ, Hellyer TJ, Brown IN, Weber JN. Clinical aspects of mycobacterial infections in HIV infection. Res Microbiol 1992;143:377-81.

2. Murcia M, Gómez JE, Alvarado F, Bustillo JG, Mendivelson E, Gómez B, et al. Frequency of tuberculous and non tuberculous mycobacteria in HIV infected patients from Bogota, Colombia. BMC Infect Dis 2001;1:21.

3. Frothingham R, Wilson KH. Molecular phylogeny of the Mycobacterium avium complex demonstrates clinically meaningful divisions. J Infect Dis 1993;169:30512.

4. Mijs W, Haas P, Rossau R, van der Laan T, Rigouts L, Portaels F, et al. Molecular evidence to support a proposal to reserve the designation Mycobacterium avium subsp. avium for bird type isolates and $M$. avium subsp. hominissuis for the human/porcine type of $M$. avium. Int J Syst Evol Microbiol 2002;52:1505-18.

5. Devallois A, Goh KS, Rastogi N. Rapid identification of mycobacteria to species level by PCR-restriction fragment length polymorphism analysis of the $h s p 65$ gene and proposition of an algorithm to differentiate 34 mycobacterial species. J Clin Microbiol 1997;35:296973 .

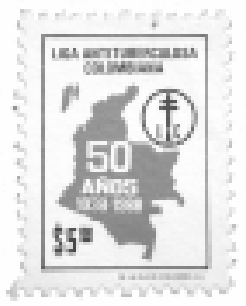

6. Reisner BS, Gatson AM, Woods GL. Use of GenProbe AccuProbes to identify Mycobacterium avium complex, Mycobacterium tuberculosis complex, Mycobacterium kansasii, and Mycobacterium gordonae directly from Bactec TB broth cultures. J Clin Microbiol 1994;32:2995-8.

7. Telenti A, Marchesi F, Balz M, Bally F, Bottger E, Bodmer T. Rapid identification of mycobacteria to the species level by polymerase chain reaction and restriction analysis. J Clin Microbiol 1993;31:175-8.

8. Devallois A, Picardeau C N, Paramasivan V, Vincent V, Rastogi N. Molecular characterization of Mycobacterium avium complex isolates giving discordant results in AccuProbe tests by PCRrestriction enzyme analysis, 16S rRNA sequencing, and DT1-DT6 PCR. J Clin Microbiol 1997;35:2767-72.

9. Leão SC, Briones MRS, Sircili MP, Balian SC, Mores $\mathbf{N}$, Ferreira-Neto JS. Identification of two novel Mycobacterium avium allelic variants by PCR-restriction enzyme analysis in pig and human isolates from Brazil. J Clin Microbiol 1999;37:2592-7.

10. Oliveira RS, Sircili MP, Ueki SYM, Telles MAS, Schinabel B, Briones MRS, et al. PCR-restriction enzyme analysis of bone marrow isolate form a human immunodeficiency virus-positive patient discloses polyclonal infection with two Mycobacterium avium strains. J Clin Microbiol 2000;38:4643-5.

11. Smole S, Mc Aleese F, Ngampasutadol J, von Reyn F, Arbeit R. Clinical and epidemiological correlates of genotypes within the Mycobacterium avium complex defined by restriction and sequence analysis of $h s p 65$. $\mathrm{J}$ Clin Microbiol 2002;40:3374-80.

12. Oliveira RS, Sircili MP, Oliveira EMD, Balian SC, Ferreira-Neto JS, Leão SC. Identification of Mycobacterium avium genotypes with distinctive traits by combination of IS1245-based restriction fragment length polymorphism and restriction analysis of $h s p 65$. J Clin Microbiol 2003;41:44-9.

13. Murcia M, León CI, De la Hoz F, Saravia J. Frecuencia de infecciones micobacterianas en pacientes $\mathrm{VIH}$ positivos que acuden a la consulta del Hospital San Juan de Dios. Biomédica 1996;16(Suppl.1):23.

14. Turenne CY, Tschetter L, Wolfe J, Kabani A. Necessity of quality-controlled $16 \mathrm{~S}$ rRNA gene sequence databases identifying nontuberculous Mycobacterium species. J Clin Microbiol 2001;39:3637-48. 